\title{
High resolution spectroscopy of Balmer-dominated shocks in the RCW 86, Kepler and SN 1006 supernova remnants ${ }^{\star}$
}

\author{
J. Sollerman ${ }^{1}$, P. Ghavamian ${ }^{2,4}$, P. Lundqvist ${ }^{1}$, and R. C. Smith ${ }^{3,4}$ \\ 1 Stockholm Observatory, Department of Astronomy, AlbaNova, 10691 Stockholm, Sweden \\ 2 Department of Physics and Astronomy, Rutgers University, 136 Frelinghuysen Rd., Piscataway, NJ 08854-8019, USA \\ ${ }^{3}$ Cerro Tololo Inter-American Observatory, Casilla 603, Chile \\ 4 Visiting Astronomer, Cerro Tololo Inter-American Observatory, National Optical Astronomy Observatories, \\ CTIO is operated by AURA, Inc. under contract to the National Science Foundation
}

Received 25 March 2003 / Accepted 22 May 2003

\begin{abstract}
We report results from high resolution optical spectroscopy of three non-radiative galactic supernova remnants, RCW 86, Kepler's supernova remnant and SN 1006. We have measured the narrow component $\mathrm{H} \alpha$ line widths in Balmerdominated filaments in RCW 86 and SN 1006, as well as the narrow component width in a Balmer-dominated knot in Kepler's SNR. The narrow component line widths measured in RCW 86 and Kepler's SNR show FWHM of 30-40 km s' ${ }^{-1}$, similar to what has been seen in other Balmer-dominated remnants. Of the remnants in our sample, SN 1006 is the fastest shock $\left(\sim 3000 \mathrm{~km} \mathrm{~s}^{-1}\right)$. The narrow component $\mathrm{H} \alpha$ and $\mathrm{H} \beta$ lines in this remnant have a $F W H M$ of merely $21 \mathrm{~km} \mathrm{~s}^{-1}$. Comparing the narrow component widths measured in our sample with those measured in other remnants shows that the width of the narrow component does not correlate in a simple way with the shock velocity. The implications for the pre-heating mechanism responsible for the observed line widths are discussed.
\end{abstract}

Key words. ISM: supernova remnants - shock waves - ISM: individual objects: SN 1006 - ISM: individual objects: RCW 86 - ISM: individual objects: Kepler

\section{Introduction}

Supernova (SN) explosions produce some of the strongest shocks in nature. At the low ISM densities $\left(n \leq 1 \mathrm{~cm}^{-3}\right)$ such shocks are often non-radiative, losing a negligible fraction of their internal energy to radiative cooling. The low density of the preshock gas makes Coulomb collisions between charged particle species very infrequent. The slow subsequent Coulomb heating in fast shocks ensures unequal temperatures of electrons and ions in the postshock flow.

Unlike a radiative shock, the optical emission from a nonradiative shock is produced entirely by collisional excitation in the ionization zone immediately behind the shock (Raymond 1991). If the preshock medium is significantly neutral, the emission from a non-radiative shock is dominated by hydrogen line emission (i.e., it is Balmer-dominated, Chevalier \& Raymond 1978). The Balmer line profiles are quite remarkable. When a non-radiative shock encounters partially neutral gas, cold hydrogen atoms overrun by the shock are collisionally excited. The radiative decay of these excited

Send offprint requests to: J. Sollerman,

e-mail: jesper@astro.su.se

* Based on observations collected at the European Southern Observatory, Paranal, Chile (ESO Programme 67.D-0579). neutrals produces narrow-component Balmer emission with a line width determined by the pre-shock temperature. On the other hand, charge exchange between cold neutrals and protons produces fast hydrogen atoms with the velocity distribution of the postshock protons. Collisional excitation of these fast neutrals produces broad Balmer line emission (Chevalier \& Raymond 1978; Chevalier et al. 1980).

Balmer-dominated shocks have been detected in five Galactic supernova remnants (SNRs): SN 1006 and Tycho's SNR (Kirshner et al. 1987; Smith et al. 1991; Ghavamian et al. 2002), Kepler's SNR (Blair et al. 1991), RCW 86 (Long \& Blair 1990; Smith 1997; Ghavamian et al. 2001) and the Cygnus Loop (Raymond et al. 1983; Hester et al. 1994; Ghavamian et al. 2001). Four remnants in the Large Magellanic Cloud also exhibit Balmer-dominated emission (Tuohy et al. 1982; Smith et al. 1991, 1994). The width of the broad component Balmer line and the broad-to-narrow flux ratio observed in the spectra of these remnants can be used to simultaneously measure the shock speed and the ratio $T_{\mathrm{e}} / T_{\mathrm{i}}$ immediately behind the shock (e.g., Ghavamian et al. 2001).

During the last ten years, high-resolution spectra of Balmer dominated shocks have revealed narrow component line widths ranging from $30 \mathrm{~km} \mathrm{~s}^{-1}$ to $50 \mathrm{~km} \mathrm{~s}^{-1}$, implying preshock temperatures of 20000-60000 K (Hester et al. 1994; 
Smith et al. 1994). This is clearly larger than the thermal width of ambient, undisturbed interstellar matter. If the narrow component widths represent thermal broadening, their large values in Balmer-dominated SNRs suggest that the ambient gas is somehow heated in a precursor by energetic particles which escape upstream and deposit their energy into the preshock gas (Hester et al. 1994; Smith et al. 1994). These particles may be cosmic rays, and such precursors are predicted by diffusive shock acceleration models (Blandford \& Eichler 1987). Alternatively, the same fast neutrals responsible for the broad component $\mathrm{H} \alpha$ emission could escape upstream and deposit their energy into the preshock gas via charge exchange and elastic collisions, thus forming a fast neutral precursor (Smith et al. 1994; Lim \& Raga 1996).

The correlation between the shock velocity and the width of the narrow component line could be a potentially important discriminant between different precursor scenarios. A preliminary study of upstream heating by fast neutrals suggests that the degree of precursor heating should be sensitive to the shock speed, degree of electron-ion temperature equilibration at the shock front and the preshock neutral fraction (Smith et al. 1994). On the other hand, it is not clear whether heating of the upstream gas by a cosmic ray precursor should have a strong dependence on the shock velocity (e.g., Hester et al. 1994; Smith et al. 1994; Boulares \& Cox 1988).

In this investigation we have obtained the first high resolution optical spectra of three Balmer-dominated Galactic supernova remnants, RCW 86, Kepler's supernova remnant and SN 1006. In Sect. 2 we present our observations and the techniques used to reduce the data. In Sect. 3 we present the obtained results and in Sect. 4 we discuss these results in conjunction with other data on nonradiative supernova shocks. Finally, the results are summarized in Sect. 5 .

\section{Observations and data reduction}

\subsection{RCW 86 and Kepler}

The galactic SNR RCW 86 was observed on 1999 June 7, using the Echelle spectrograph at the $\mathrm{f} / 7.8$ focus of the $4 \mathrm{~m}$ telescope of Cerro Tololo Inter-American Observatory. The T2K detector was connected to the Red Long Camera and binned by two in the spatial dimension, giving a plate scale of $0 .{ }^{\prime \prime} 53 \mathrm{pixel}^{-1}$. The decker was set to the open position, giving a spectrograph slit length of 5.68. However, vignetting limited the useful length of slit to around 3!68. The 79 lines $\mathrm{mm}^{-1}$ Echelle grating was used for the observations, with the cross disperser replaced by a flat mirror. The width of the spectrograph slit was 1."3, which combined with the grating arrangement yielded a spectral resolution of $11.2 \mathrm{~km} \mathrm{~s}^{-1}$. The spectra were centered on the $\mathrm{H} \alpha$ line, while all other emission lines were excluded by inserting an $\mathrm{H} \alpha$ filter $\left(\lambda_{\mathrm{c}}=6563 \AA, \Delta \lambda=75 \AA\right)$. Wavelength calibration was performed using ThAr lamp spectra interleaved with object exposure frames.

The spectroscopic observations of RCW 86 targeted three different Balmer-dominated filaments of the remnant (Fig. 1). The atmospheric seeing during the RCW 86 observations ranged from $11^{\prime \prime} 5$ to $2^{\prime \prime}$. Of these filaments, the brightest is the one located in the southwestern region of the remnant, where the Balmer-dominated shocks lie close to bright radiative shocks (Rosado et al. 1996; Smith 1997; Ghavamian et al. 2001). Filaments located in the NW and W portions of RCW 86 are nearly twice as faint as those in the SW. However, moderate resolution spectra show that the widths of the broad component $\mathrm{H} \alpha$ lines are comparable in all three regions, ranging from $560 \mathrm{~km} \mathrm{~s}^{-1}$ in the SW (Ghavamian et al. 2001) to around $600 \mathrm{~km} \mathrm{~s}^{-1}$ in the NW/W (Ghavamian 1999). The procedures for targeting the Balmer-dominated shocks and acquisition of the Echelle spectra were as follows:

(1) RCW $86 \mathrm{NW}$ - The spectrograph slit was centered on a star located at $\alpha=14: 40: 23.6, \delta=-62: 16: 46.9$ (2000), then offset $1^{\prime \prime} \mathrm{S}$ and rotated to PA $=240^{\circ}$. Five frames were acquired at this position for a total integration time of $4500 \mathrm{~s}$.

(2) RCW $86 \mathrm{~W}$ - The spectrograph slit was centered on a star located at $\alpha=14: 40: 38.8, \delta=-62: 24: 35.4$ (2000), then offset $1^{\prime \prime} \mathrm{S}$ and rotated to $\mathrm{PA}=225^{\circ}$. Five frames were acquired at this position for a total integration time of $4500 \mathrm{~s}$.

(3) RCW $86 \mathrm{SW}-$ The spectrograph slit was centered on a star located at $\alpha=14: 41: 09.4, \delta=-62: 43: 57.0$ (2000), then rotated to $\mathrm{PA}=308.7^{\circ}$. Five frames were acquired at this position for a total integration time of $3000 \mathrm{~s}$.

High resolution spectra of Kepler's SNR were obtained on the same night as the RCW 86 observations. During this time the seeing had degraded, ranging between $3^{\prime \prime}$ and $4^{\prime \prime}$. The Kepler observations targeted the knotty structures in the remnant interior identified as Knots D49 \& D50 (D'Odorico et al. 1986; Blair et al. 1991). The slit was oriented at PA $=290^{\circ}$ and placed so that the upper portion of the slit intersected the bright radiative knots along the NW part of Kepler's SNR, with fainter radiative knots seen near the middle of the slit and the pure Balmer-dominated Knots D49 \& D50 seen in the lower half of the slit (Fig. 1). The spectrograph slit was centered on the position $\alpha=17: 30: 40.5, \delta=-21: 29: 23.0$ (2000), then rotated to $\mathrm{PA}=290^{\circ}$. Five frames were acquired at this position for a total integration time of $4500 \mathrm{~s}$.

The RCW 86 and Kepler's SNR data were reduced in IRAF using the standard processes of overscan and bias correction, dark count correction and flat fielding. Wavelength solutions were computed at evenly spaced intervals along the slit and used to untilt the dispersion axes of the spectra. Finally, the background was subtracted from each two-dimensional spectrum using sky emission adjacent to each target along the slit.

\section{2. $S N 1006$}

The observations of SN 1006 were obtained with the Ultraviolet and Visual Echelle Spectrograph (UVES). This instrument is located at one Nasmyth focus on the second unit telescope (Kueyen) of the Very Large Telescope (VLT) on Paranal, Chile. UVES is a high-resolution two-arm cross-dispersed Echelle spectrograph, where both arms can be operated separately or simultaneously using a dichroic beam-splitter.

The VLT/UVES observations were conducted in visitor mode on June 13, 2001. As we were primarily interested in $\mathrm{H} \alpha$ and $\mathrm{H} \beta$ we chose to observe in the red arm only. 

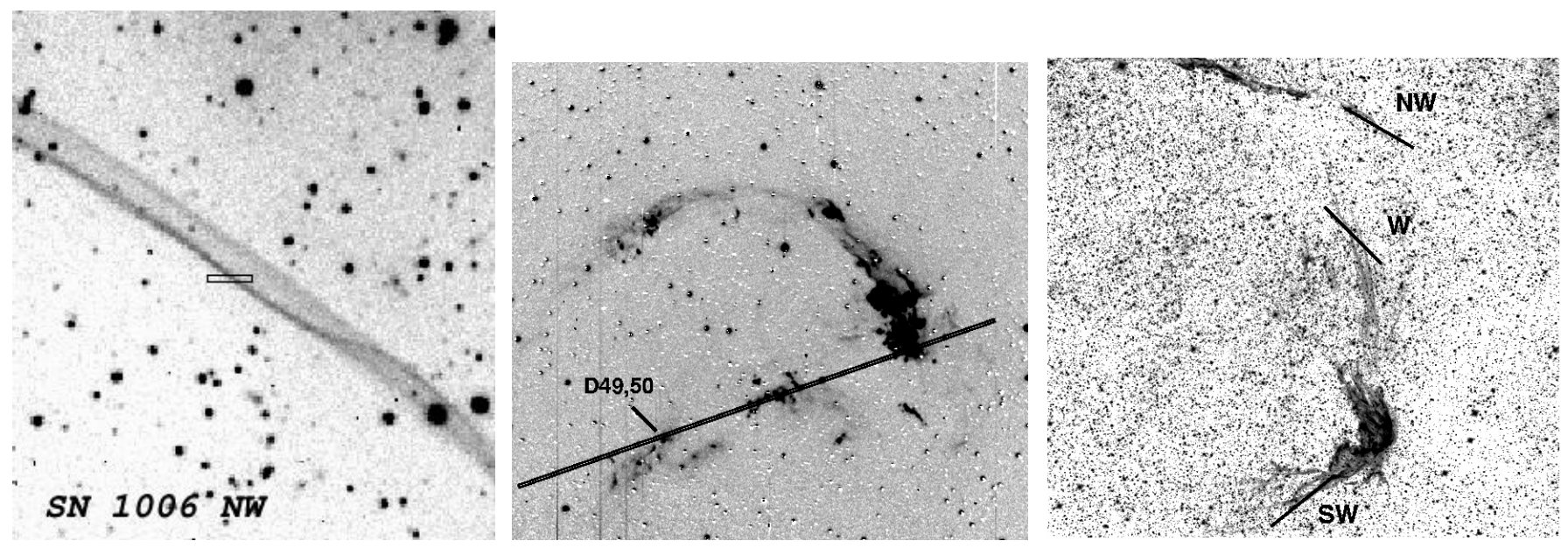

Fig. 1. The positions of the slits used for our observations of the three supernova remnants. The leftmost image (from Winkler \& Long 1997) shows SN 1006 with the 12" UVES slit indicated. North is up and east to the left in all three images. The middle image (from Blair et al. 1991) shows the Kepler supernova remnant, indicating our slit position and the knots D49 \& D50. The slit length is 5'.68. The rightmost image (from Smith 1997) shows our three slit-positions used for RCW 86. Slit lengths are again 5'.68.

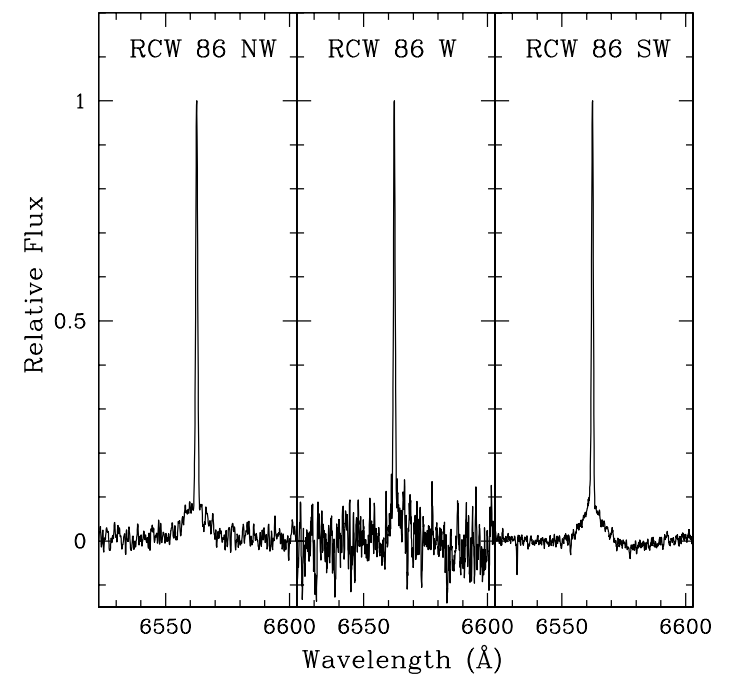

Fig. 2. One-dimensional sky-subtracted spectra of the NW, W and SW Balmer-dominated shocks in RCW 86. The middle spectrum has been smoothed over $0.8 \AA$, while the NW and SW spectra have been smoothed over $0.4 \AA$. The broad component is clearly detected in the NW and SW spectra, with the faint W spectrum exhibiting weak evidence of a broad component.

With Cross-disperser \#3 and the order sorter filter SHP700 this set-up ${ }^{1}$, centered on $5800 \AA$, covers the wavelength region 4760-6840 ̊.

UVES has a short slit with a length of $12^{\prime \prime}$. We used a slit width of 1". 8 positioned as shown in Fig. 1. The red arm is equipped with a mosaic of two $2 \mathrm{k} \times 4 \mathrm{k}$ CCDs, with a scale of 0.022-0.026 $\AA$ pixel $^{-1}$ and a spatial scale of 0. ' 18 pixel $^{-1}$. The position shown in Fig. 1 was observed in 7 exposures of $15 \mathrm{~min}$ each, in total $6300 \mathrm{~s}$. The airmass was secz = $1.16-1.48$ and the seeing varied between $1 .{ }^{\prime} 1$ and $1{ }^{\prime \prime} 5$.

\footnotetext{
${ }^{1}$ See http://www.eso.org/instruments/uves/ for details on the instrument and observational technique.
}

The spectra were interactively reduced using the UVES-pipeline $^{2}$ (version 1.2.0) as implemented in MIDAS. The reduction separates the two CCDs and performs bias subtraction and flat-fielding of the data using calibration frames obtained in the morning.

Wavelength calibration was done by comparison to ThAr arc lamps. More than 1000 spectral lines were fitted for both of the CCDs, with an rms of better than $0.008 \AA$. The resolution for arc-lines with the 1. .' 8 slit is $\sim 13 \mathrm{~km} \mathrm{~s}^{-1}$, or $0.29 \AA$ at the position of $\mathrm{H} \alpha$.

The pipeline finally extracts the spectrum of the reduced frames. We averaged the signal over 5"' 5 along the slit, and subtracted the skylines from the region at the very end of the slit.

\section{Results}

\subsection{RCW 86}

The spectra of all three observed positions in RCW 86 appear in Fig. 2. The NW and W spectra were obtained by summing $15^{\prime \prime}$ and $10^{\prime \prime}$ of emission from each filament, respectively, while the emission from the bright SW filament was summed over 2." 5 . The broad component is detected in all three spectra. Although a two-component fit was performed for each of the spectra, the narrow component line in each case is bright and well separated in width from the broad component. This makes the estimated narrow component width rather insensitive to the inclusion of a second component in the fit. The measured narrow component $F W H M$ widths were $40 \pm 2 \mathrm{~km} \mathrm{~s}^{-1}, 32 \pm 5 \mathrm{~km} \mathrm{~s}^{-1}$ and $32 \pm 2 \mathrm{~km} \mathrm{~s}^{-1}$ for the NW, W and SW regions, respectively. Due to the large dispersion of the Echelle spectra, the broad component wings are not well detected, and our fits yield only lower limits on the broad component widths. The fitted values are $\gtrsim 450 \mathrm{~km} \mathrm{~s}^{-1}$, consistent with measurements at lower

\footnotetext{
${ }^{2}$ http://www. eso.org/observing/dfo/quality/
} 


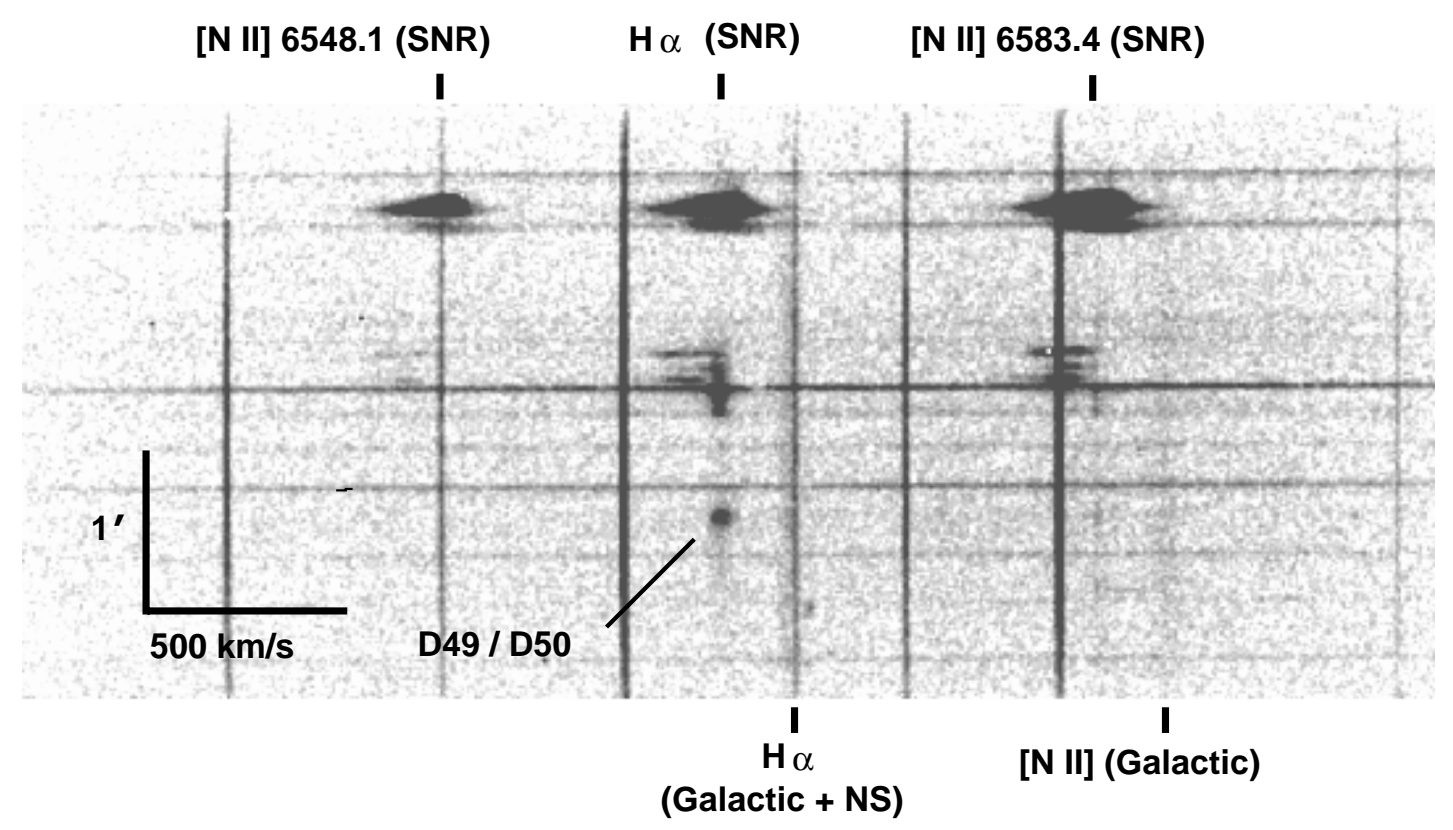

Fig. 3. The two-dimensional spectrum of Kepler's SNR, shown smoothed with a 1 pixel Gaussian and without subtraction of night sky lines. The Balmer-dominated knots D49 \& D50 are marked, along with the corresponding [N II] emission lines from the SNR and the Galactic background. The remaining bright lines are night sky (NS) emission features. The teardrop shaped structures at the top of the slit and the fainter structures near the middle of the slit are radiative shocks in clumpy, nitrogen-rich material associated with Kepler's SNR.

resolution (Long \& Blair 1990; Ghavamian 1999; Ghavamian et al. 2001).

\subsection{Kepler's SNR}

In the two-dimensional spectrum of Kepler's SNR (Fig. 3) a number of knots appear along the length of the slit exhibiting $\mathrm{H} \alpha$ and [N II] line emission. The bottom knot corresponds to knots D49 \& D50 tabulated by D'Odorico et al. (1986) and identified as Balmer-dominated shocks by Blair et al. (1991). Most of the structures seen along the Echelle slit exhibit a systemic blueshift of $185 \mathrm{~km} \mathrm{~s}^{-1}$.

In Fig. 4 we present the spectrum of D49 \& D50 with the night sky lines included to illustrate the large width of the $\mathrm{H} \alpha$ narrow component. The width of the narrow $\mathrm{H} \alpha$ component in knots D49 \& D50 is $42 \pm 3 \mathrm{~km} \mathrm{~s}^{-1}$ after correction for instrumental broadening. The faint, broad hump of emission observed in Fig. 4 is broad component $\mathrm{H} \alpha$ emission $\left(v_{F W H M} \approx 1800 \mathrm{~km} \mathrm{~s}^{-1}\right.$ according to Blair et al. 1991) from knots D49 \& D50. At the high resolution of these observations, it is difficult to measure the parameters of this component precisely, but both the centroid and broad-to-narrow flux ratio are consistent with those reported in Blair et al. (1991; $\sim 6571 \AA$ and 1.2 , respectively)

The non-sky subtracted spectrum of knots D49 \& D50 (Fig. 4) exhibits two faint [N II] $\lambda 6583$ features, one seen at close to zero velocity and one blueshifted to $185 \mathrm{~km} \mathrm{~s}^{-1}$, the systemic speed of Kepler's SNR. The line at zero velocity is rather broad $\left(\sim 100 \mathrm{~km} \mathrm{~s}^{-1}\right)$; the two-dimensional spectrum (Fig. 3) shows that this line is of nearly uniform surface brightness and is seen along the full length of the unvignetted spectrograph slit. The most likely origin for the broad [N II] is Galactic emission. Its large width is likely due to a blend of multiple [N II] lines from clouds along the line of sight.

More interesting is the narrow, blueshifted [N II] $\lambda 6583$ line seen in the spectrum of knots D49 \& D50. From the twodimensional spectrum (Fig. 3) it is clear that this [N II] emission arises from knots D49 \& D50. After correction for instrumental broadening, we find a width of $20 \pm 2 \mathrm{~km} \mathrm{~s}^{-1}$ for the [N II] $\lambda 6583$ line in knots D49 \& D50. The presence of this line in $2000 \mathrm{~km} \mathrm{~s}^{-1}$ Balmer-dominated shocks is further discussed below (Sect. 4.3).

\section{3. $S N 1006$}

In Fig. 5 we show the fully reduced $\mathrm{H} \alpha$ line from the part of SN 1006 shown in Fig. 1. The narrow Balmer line is indeed resolved. We measure the FWHM by a Gaussian fit to be $24 \mathrm{~km} \mathrm{~s}^{-1}$. This is clearly broader than the surrounding skylines, which show a $F W H M$ of about $12 \mathrm{~km} \mathrm{~s}^{-1}$. Thus, correcting the width of the $\mathrm{H} \alpha$ line for the instrumental resolution, we arrive at an intrinsic width of $21 \mathrm{~km} \mathrm{~s}^{-1}$. The inset in Fig. 5 shows a Gaussian fit $10 \%$ wider and narrower, and from this we estimate the error in the narrow component $F W H M$ to be $\lesssim 3 \mathrm{~km} \mathrm{~s}^{-1}$.

Apart from $\mathrm{H} \alpha$, and consistent with the medium-resolution spectra of Ghavamian et al. (2002), the only other line detected in $\mathrm{SN} 1006$ is $\mathrm{H} \beta$. While this line is more noisy, the width can nevertheless be measured by a Gaussian fit. The width corrected for instrumental resolution is the same as for $\mathrm{H} \alpha$, i.e., $21 \mathrm{~km} \mathrm{~s}^{-1}$. The nearby skylines have widths of slightly more than $12 \mathrm{~km} \mathrm{~s}^{-1}$, consistent with the resolution obtained in the arc lamp spectral lines. 


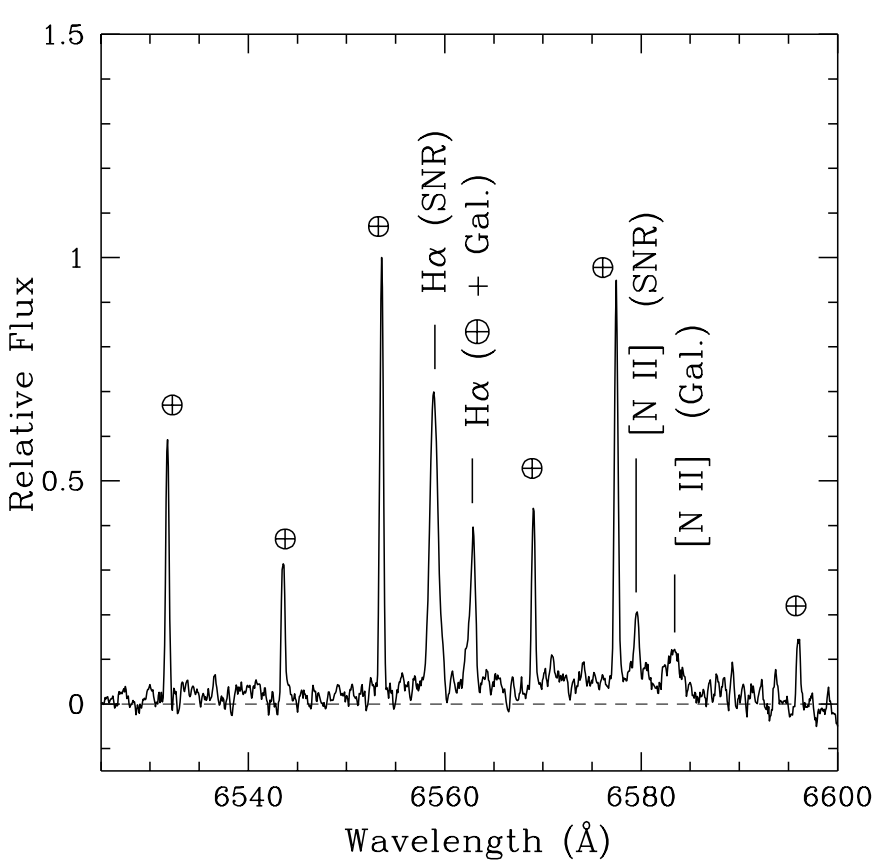

Fig. 4. The extracted spectrum of Balmer-dominated knots D49 \& D50 in Kepler's SNR, smoothed over $0.4 \AA$. Night sky lines have been left in and are marked. There are three emission lines associated with the knots: narrow $\mathrm{H} \alpha\left(42 \mathrm{~km} \mathrm{~s}^{-1}\right)$ and broad $\mathrm{H} \alpha\left(\sim 2000 \mathrm{~km} \mathrm{~s}^{-1}\right)$ lines and a narrow [N II] $\lambda 6583$ line $\left(20 \mathrm{~km} \mathrm{~s}^{-1}\right)$. All three lines are blueshifted $185 \mathrm{~km} \mathrm{~s}^{-1}$ from zero velocity. The unshifted, broad [N II] line seen near $6583 \AA$ is Galactic emission (see discussion in text).

\section{Analysis and discussion}

\subsection{Comparison to other Balmer dominated supernova remnants}

The narrow component in several other Balmer dominated shocks have been spectroscopically resolved. Smith et al. (1994) studied four remnants in the LMC, and found narrow component widths of $30-50 \mathrm{~km} \mathrm{~s}^{-1}$. The same year, Hester et al. (1994) investigated the Balmer dominated parts of the Cygnus loop and found similar values for the narrow components. We have included our five narrow component width measurements in Table 1, along with a listing of prior measurements in other SNRs for comparison.

The shocks in Table 1 of RCW 86, Cygnus and Tycho were modeled in detail by Ghavamian et al. (2001), and SN 1006 by Ghavamian et al. (2002). The shock velocities for the three LMC remnants in Table 1 were estimated by Raymond (2001) based on the observations by Smith et al. (1991) and extrapolating the relation between shock velocity and equilibration.

The broad component width measured for knots D49 \& D50 in Kepler's supernova remnant by Blair et al. (1991) is $1700-2000 \mathrm{~km} \mathrm{~s}^{-1}$, similar to the value measured for Knot $\mathrm{g}$ in Tycho's SNR (Smith et al. 1991; Ghavamian et al. 2001). However, the broad-to-narrow ratio for D49 \& D50 is 1.2, nearly twice as large as that of Tycho Knot $\mathrm{g}$. Based on our non-radiative shock models (Ghavamian et al. 2001, 2002) we estimate that a shock speed of $2000-2500 \mathrm{~km} \mathrm{~s}^{-1}$ is appropriate for Kepler knots D49 \& D50. This is assuming a low electronion equilibration at the shock front, $T_{\mathrm{e}} / T_{\mathrm{p}}<0.2$. For the
Cygnus Loop it was not exactly the same region of the SNR measured for the broad and narrow components. For RCW 86, the broad component widths measured from low resolution spectra are nearly identical in all three regions (Ghavamian 1999; Ghavamian et al. 2001), so we have assumed that the shock speeds are also equal.

We see that the widths of the narrow components determined for RCW 86 and Kepler are very similar to those found in other supernova remnants. In fact, the main conclusion to be drawn from Table 1 is that the narrow component widths remain remarkably constant over a factor of 10 in estimated shock velocity. The widths we measure for RCW 86, Kepler's SNR and SN 1006 strengthen this conclusion. The noticeable drop in narrow component width at the highest shock speed, that of SN 1006, is still only a factor of $\sim 2$ lower than the largest widths measured thus far.

In Fig. 6 we have plotted the data from Table 1. If we disregard the data from SN 1006 (discussed below), a weak trend for broader narrow components in faster shocks can be seen. A simple linear chi-square $\left(\chi^{2}\right)$ fit that only allows for errors in the narrow component $F W H M$ gives a slope of $5.2( \pm 1.6) \mathrm{km} \mathrm{s}^{-1}$ for every $1000 \mathrm{~km} \mathrm{~s}^{-1}$ increase in shock velocity. This fit has a reduced $\chi^{2}$ of 1.6. A constant line does not give a good fit. It gives a reduced $\chi^{2}$ of 2.7 , which is rejected at a $2.8 \sigma$ level. Thus if these error bars are to be interpreted formally (and if SN 1006 can be ignored), a preshock heating mechanism with modest but positive velocity dependence is favored. It remains to be seen if detailed modeling of cosmic ray pre-heating (e.g., Boulares \& Cox 1988) can account for this trend.

However, the alternative scenario may be even more problematic. Though some of the broad component neutrals undoubtedly cross into the preshock region, their energy distribution and number density are sensitive to a number of parameters. A neutral precursor model that favors a strong (roughly quadratic, Smith et al. 1994) dependence on the shock velocity is clearly not consistent with the relatively small range of narrow component widths among the Balmer-dominated remnants summarized in Table 1. This was noted already by Smith et al. (1994).

\subsection{The narrow component line width in SN 1006}

As seen in Table 1 and in Fig. 6, the narrowest of the lines is the one in SN 1006. The resolved narrow component of the Balmer lines has a width of merely $21 \mathrm{~km} \mathrm{~s}^{-1}$. This corresponds to a kinetic temperature of $\sim 9500 \mathrm{~K}$. The shock velocity of SN 1006 has recently been accurately determined when a deep spectrum of SN 1006 was obtained by Ghavamian et al. (2002). Summing up emission along 51" of their slit, they were able to detect even He I 26678 . These observations, together with a detailed non-radiative shock model, confirmed a very low degree of electron-proton equilibration at the shock front, $T_{\mathrm{e}} / T_{\mathrm{p}} \leq 0.07$, and the shock speed was determined to be $2890 \pm 100 \mathrm{~km} \mathrm{~s}^{-1}$.

Our data thus indicate that the fastest of the Balmerdominated shocks in our sample displays a line width close to that expected for the undisturbed, warm component of the ISM 


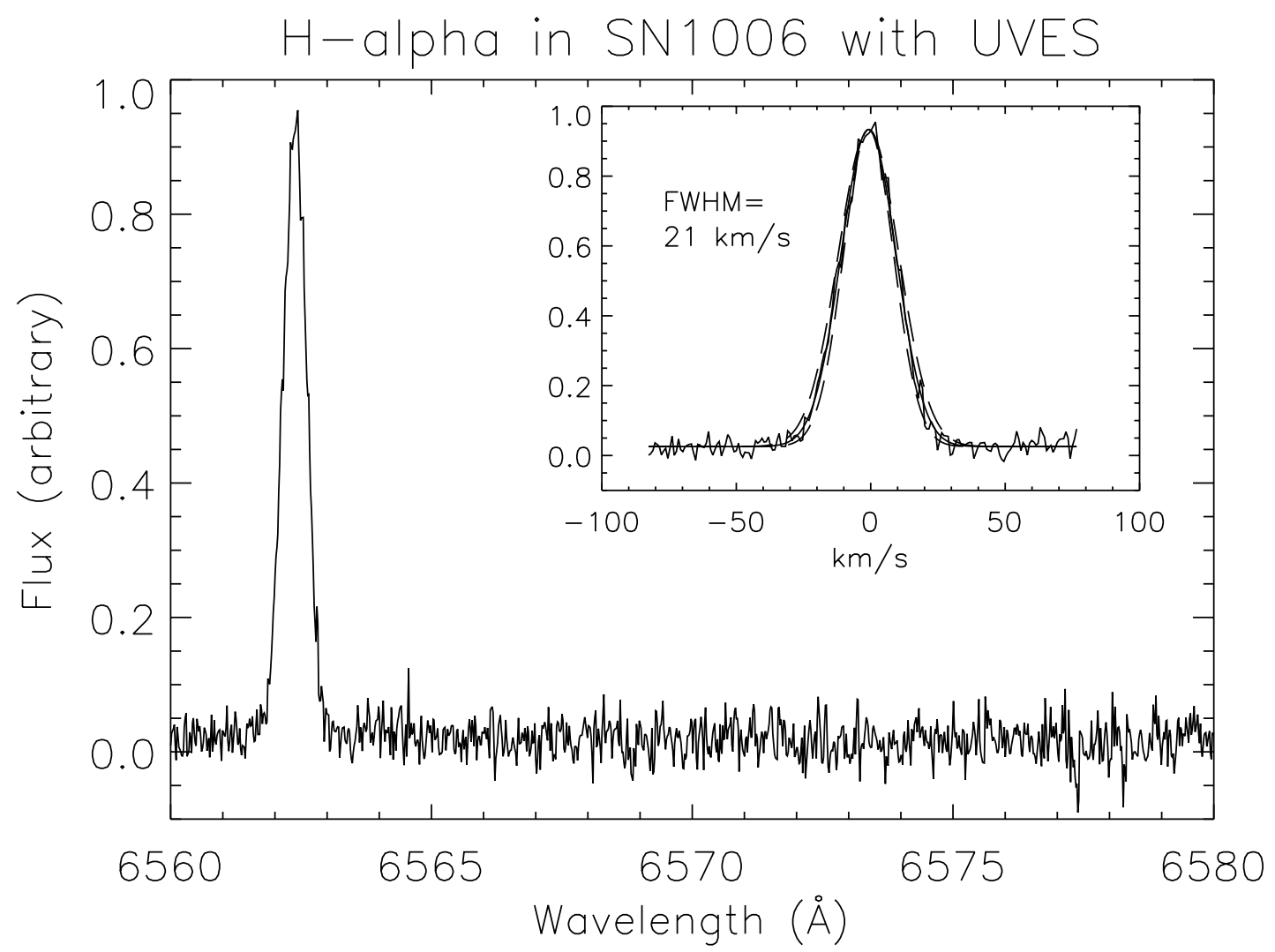

Fig. 5. The narrow $\mathrm{H} \alpha$ line detected in SN 1006. The inset shows the line in velocity-space, with the best Gaussian fit of $F W H M=21 \mathrm{~km} \mathrm{~s}^{-1}$ (full line). The broken lines indicate Gaussian fits with $F W H M$ which are $10 \%$ wider and narrower. Zero velocity refers to $6562.4 \AA$. The broad component of $\mathrm{H} \alpha$ (cf. Ghavamian et al. 2002) is not detected in these high resolution observations.

Table 1. Balmer dominated supernova remnants.

\begin{tabular}{lcccc}
\hline \hline SNR & $\begin{array}{c}\text { Shock velocity } \\
\left(\mathrm{km} \mathrm{s}^{-1}\right)\end{array}$ & $\begin{array}{c}\text { Narrow component } \\
F W H M\left(\mathrm{~km} \mathrm{~s}^{-1}\right)\end{array}$ & References & $\begin{array}{c}\kappa \\
\left(\mathrm{cm}^{2} \mathrm{~s}^{-1}\right)\end{array}$ \\
\hline Cygnus Loop & $300-400$ & $28-35$ & GRSH01,HRB94 & \\
RCW 86 SW & $580-660$ & $32 \pm 2$ & GRSH01, This work & $<3.3 \times 10^{26} / n_{\mathrm{e}}$ \\
RCW 86 W & $580-660$ & $32 \pm 5$ & This work & $<1.2 \times 10^{27} / n_{\mathrm{e}}$ \\
RCW 86 NW & $580-660$ & $40 \pm 2$ & This work & $<1.5 \times 10^{25} / n_{\mathrm{e}}$ \\
Kepler D49 \& D50 & $2000-2500$ & $42 \pm 3$ & BLV91,This work & $<1.7 \times 10^{27} / n_{\mathrm{e}}$ \\
0505-67.9 & $440-880$ & $32-43$ & SKBW91, R01,SRL94 & \\
0548-70.4 & $700-950$ & $32-58$ & SKBW91,R01,SRL94 & \\
0519-69.0 & $1100-1500$ & $39-42$ & SKBW91, R01,SRL94 & \\
0509-67.5 & - & $25-31$ & SRL94 & \\
Tycho & $1940-2300$ & $44 \pm 4$ & GRSH01, GRHB00 & \\
SN 1006 & $2890 \pm 100$ & $21 \pm 3$ & GWRL02, This work & \\
\hline
\end{tabular}

(Ferriére 2001; Raymond 2001). Whatever mechanism broadens the narrow component in slower shocks is likely to operate also at higher Mach numbers. But these data do not favor a model where the pre-heating is simply correlated with the shock velocity, as suggested for fast neutrals (Smith et al. 1994, but see also Hester et al. 1994).
The process heating the pre-shock gas in SN 1006 appears to be less efficient than in the other SNRs listed in Table 1. Detailed modeling of the energy deposition of cosmic rays or neutrals passing upstream through the shock is still in its infancy (e.g., Boulares \& Cox 1988; Lim \& Raga 1996) and is outside the scope of this paper. 


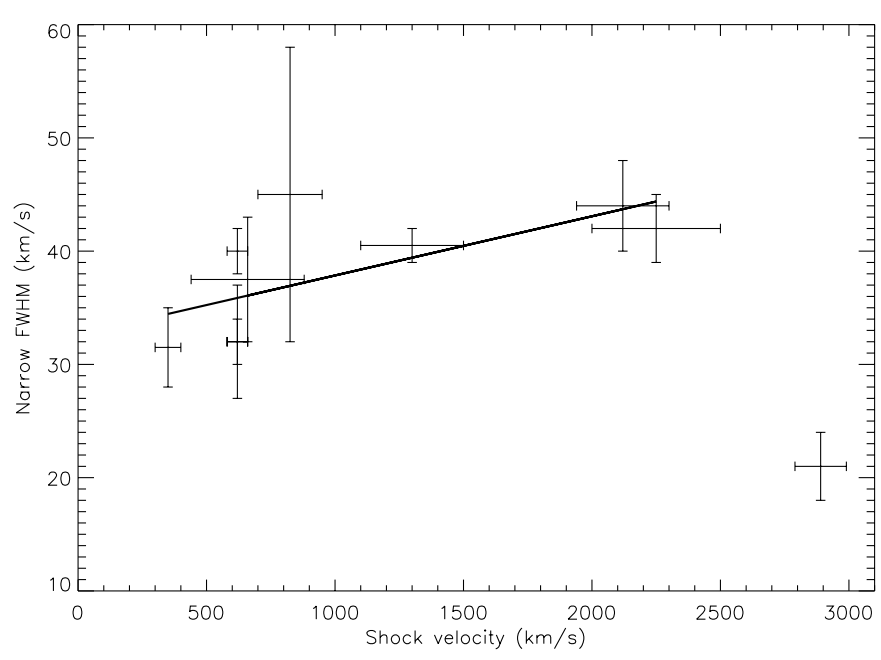

Fig. 6. Shock velocity versus the $F W H M$ of the narrow component of the observations listed in Table 1, except for SNR 0509-67.5 for which there is no estimated shock velocity. The line shows the best linear fit to the data, as discussed in the text.

It is unclear whether our result for the SN 1006 Balmer filaments reflects a general trend of sharply declining narrow component line width at high shock speeds $\left(\simeq 3000 \mathrm{~km} \mathrm{~s}^{-1}\right)$ or whether the small line width is caused by some unique, unknown property of the SN 1006 blast wave.

It is interesting to note that also SNR 0509-67.5 displays a rather narrow component (Table 1). In fact, Smith et al. (1994) measured $25 \pm 3 \mathrm{~km} \mathrm{~s}^{-1}$ for the central and eastern rims. This is the one LMC remnant whose broad component $\mathrm{H} \alpha$ width has eluded detection (Tuohy et al. 1982; Smith et al. 1991; Smith et al. 1994). Interestingly, the limit on the blast wave speed derived by Tuohy et al. (1982) for SNR 0509-67.5 is $\geq 3600 \mathrm{~km} \mathrm{~s}^{-1}$, i.e., similar to the speed of $3000 \mathrm{~km} \mathrm{~s}^{-1}$, measured for SN 1006. In fact, speculations of the narrow component width in this remnant were already forwarded by Smith et al. (1994). However, it is probably premature to use these small widths of SNR 0509-67.5 in conjunction with our SN 1006 results to argue for a high shock velocity cutoff in narrow component line widths in Balmer-dominated shocks. In the western rim of the same supernova remnant, Smith et al. (1994) measure a narrow component width of $31 \pm 2 \mathrm{~km} \mathrm{~s}^{-1}$, which is similar to the values seen in many other Balmer-dominated remnants. A more reliable estimate of the blast wave speed of SNR 0509-67.5 will be required to confirm the presence of a high shock velocity cutoff. Measurements of the width of the narrow component of $\mathrm{H} \alpha$ at more positions within SN 1006 could also be useful, to investigate whether the narrow width is sensitive to geometry or local pre-shock conditions.

\subsection{Narrow [N II] emission in the Balmer dominated Knots in Kepler's SNR}

In the case of Kepler's SNR, the detection of [N II] from Balmer-dominated shocks may have some bearing on the precursor question. With a broad component $\mathrm{H} \alpha$ width of $1800 \mathrm{~km} \mathrm{~s}^{-1}$ (Blair et al. 1991), the inferred shock speed in the knots is far too high for the onset of postshock cooling. Therefore, the [N II] emission from knots D49 \& D50 cannot be produced in a cooling zone as is seen in partially radiative shocks in RCW 86 (Long \& Blair 1990). Further evidence against a cooling zone origin for the [N II] is the lack of [S II] and [O III] emission from knots D49 \& D50 (Blair et al. 1991). The width of the [N II] line observed from Knots D49 \& D50 indicates that the nitrogen emission cannot come from the immediate postshock ionization zone either, since for shock speeds $\sim 2000 \mathrm{~km} \mathrm{~s}^{-1}$, nitrogen ions would be raised to temperatures exceeding $5 \times 10^{7} \mathrm{~K}$ by collisionless heating at the shock front.

The remaining possibility is that the observed [N II] emission is generated ahead of the Balmer-dominated shocks in knots D49 \& D50, in the precursor. The difference between the narrow component $\mathrm{H} \alpha$ width and the [N II] line width is interesting. If the [N II] line width is thermal, it would indicate that the nitrogen ions ahead of the shock are heated to $\sim 120000 \mathrm{~K}$, nearly three times hotter than the hydrogen atoms. This would be difficult to explain with a fast neutral precursor because in that case the energy is deposited primarily into the $\mathrm{H}$ atoms. In addition, there is little time for Coulomb collisions to communicate the heating to the $\mathrm{N}$ ions before they are all overtaken by the shock. Therefore, it is likely that the $20 \mathrm{~km} \mathrm{~s}^{-1}$ broadening of the [N II] line is nonthermal. A nonthermal broadening mechanism is readily found in the Alfvèn wave activity expected in a cosmic ray precursor. Such waves follow a spectrum of amplitudes (Blandford \& Eichler 1987; Smith et al. 1994) which can simultaneously heat ions with a range of masses. Therefore, the large [N II] line width in knots D49 \& D50 may be indirect evidence of a cosmic ray precursor. Note that this does not disprove the existence of a fast neutral precursor, but implies that such a mechanism alone is unlikely to account for the observations. The fact that [N II] is detected here but not in Balmer-dominated shocks of comparable speeds in other SNRs (such as Tycho's SNR, Smith et al. 1991; Ghavamian et al. 2001) may be due to an overabundance of nitrogen inferred for the knotty material around Kepler's SNR (Leibowitz \& Danziger 1983; Blair et al. 1991).

\subsection{Limits on the diffusion coefficient of precursor particles}

The narrow component line widths measured in RCW 86 and Kepler's SNR can be used to place a limit on the cosmic ray diffusion coefficient $\kappa$ in these remnants. Assuming that the line widths represent thermal broadening, an upper limit on the precursor width is obtained by requiring that it must be small enough to avoid complete ionization of neutral $\mathrm{H}$ before it enters the shock (Hester et al. 1994; Smith et al. 1994). We have refrained from making the same analysis for the fast shock in SN 1006, since for a preshock temperature of $\sim 9500 \mathrm{~K}$ the ionization argument can be questioned. The limit on the diffusion coefficient is then $\kappa<v_{\mathrm{S}}^{2} / n_{\mathrm{e}} q_{\mathrm{i}}\left(T_{\mathrm{e}}\right)$, where $n_{\mathrm{e}}$ is the preshock electron density and $q_{\mathrm{i}}\left(T_{\mathrm{e}}\right)$ is the ionization rate coefficient at the preshock electron temperature $T_{\mathrm{e}}$. We have 
computed the limits on $\kappa$ using the fits of Janev et al. (1987) to $q_{\mathrm{i}}$, the shock velocity estimates from Blair et al. (1991) for the Balmer-dominated knots in Kepler's SNR, and the shock velocity estimates of Ghavamian et al. (2001) for RCW 86. The results appear in Table 1. Variations in the preshock electron density due to variations in preshock ionization fraction and total density can cause variations in $\kappa$ by an order of magnitude. However, the diffusion coefficients derived here are smaller than the value $10^{29} \mathrm{~cm}^{2} \mathrm{~s}^{-1}$ expected for the galactic cosmic ray population (Ginzburg \& Syrovatskii 1964). Therefore, the coefficients derived here are at least consistent with the existence of a cosmic ray population confined to a region close to the shock front by Alfvén wave turbulence.

\subsection{The kinematic distance of RCW 86}

One source of recent debate regarding RCW 86 has been the possible association of the remnant with a neighboring OB stellar association. Rosado et al. (1996) performed Fabry-Perot spectroscopy of the bright radiative shocks in the SW portion of the remnant. They used the fitted line profiles to estimate the systemic velocity of RCW 86, and then used Galactic rotation curve parameters to estimate the distance to the remnant. We can redo the same analysis. In fact, our high spectroscopic resolution allows us to better constrain the velocity centroid of the narrow component. Moreover, the neutral gas producing the narrow $\mathrm{H} \alpha$ line is not affected by the passage of the blast wave shock (e.g., Chevalier \& Raymond 1978), so the measured line center more accurately reflects the systemic velocity of the local ambient gas than the line center measured from the radiative shocks. Following Rosado et al. (1996) we convert the observed radial velocity to a velocity relative to the LSR, then use $V_{\mathrm{LSR}}$ to compute a kinematic distance to RCW 86 from the fits of Brand \& Blitz (1993) to the Galactic rotation curve. In our analysis we assume that the galactocentric distance $R_{0}=8.5 \mathrm{kpc}$ for the Sun and that the angular speed $\omega_{0}=25.9 \mathrm{~km} \mathrm{~s}^{-1} \mathrm{kpc}^{-1}$ for the solar neighborhood (Brand \& Blitz 1993).

Using the line profile fits for the SW, W and NW blast wave spectra of RCW 86, we obtained the radial velocity from each spectrum using velocity centroids of the narrow component lines. From the wavelengths of the night sky $\mathrm{OH}$ and $\mathrm{H} \alpha$ lines in the 1D spectra, we estimate that our measured radial velocities for the narrow blast wave $\mathrm{H} \alpha$ lines were accurate to within $2 \mathrm{~km} \mathrm{~s}^{-1}$. Transforming the velocities to LSR values, we find that $V_{\mathrm{LSR}}=-33 \pm 3 \mathrm{~km} \mathrm{~s}^{-1}$, for the $\mathrm{SW}, \mathrm{W}$ and $\mathrm{NW}$ shocks. Using the rotation curves of Brand \& Blitz (1993), we find that the distance to RCW 86 implied by $V_{\mathrm{LSR}}$ in the Balmerdominated shocks is $2.3 \pm 0.2 \mathrm{kpc}$. This is in excellent agreement with the value of $V_{\mathrm{LSR}}=-33.2 \pm 4.5 \mathrm{~km} \mathrm{~s}^{-1}$, determined by Rosado et al. (1996), and within the errors our distance estimate of $2.3 \pm 0.2 \mathrm{kpc}$ agrees with the value of $2.8 \pm 0.4$ from Rosado et al. (1996). A distance of $2.3 \pm 0.2 \mathrm{kpc}$ places RCW 86 in close proximity to the $\mathrm{OB}$ association stars catalogued by Lyngå (1964). From photometry of stars in the association, Westerlund (1969) and Moffat \& Cameron (1999) estimated a distance of 2-2.5 kpc for the association.

\section{Summary}

We have added five more measurements of the narrow components in non-radiative supernova shocks. The measurements in RCW 86 and in Kepler's SNR show FWHM of $\sim 30-40 \mathrm{~km} \mathrm{~s}^{-1}$, similar to what is seen in other non-radiative Balmer-dominated shocks. The extended sample show only a weak trend for increasing $F W H M$ of the narrow lines with increasing shock velocity. The exception is the fastest shock in our sample, that in the NW limb of SN 1006. The resolved $\mathrm{H} \alpha$ and $\mathrm{H} \beta$ lines have a $F W H M$ of only $21 \pm 3 \mathrm{~km} \mathrm{~s}^{-1}$. This is lower than measured in any other Balmer dominated supernova remnant, and disfavors any precursor mechanism which simply correlates the shock velocity with the degree of pre-heating.

We have also made a first measurement of the narrow component width in a Balmer-dominated knot (Kepler's SNR), where we have also detected faint [N II] $\lambda 6583$ emission. The [N II] line width is significantly larger than expected for thermal broadening. We argue that the $[\mathrm{N} \mathrm{II}]$ line width is caused by broadening from Alfvèn wave turbulence in a cosmic ray precursor.

Finally, we have estimated a distance of $2.3 \pm 0.2 \mathrm{kpc}$ to $\mathrm{RCW} 86$, which places it at the same position as a known OB association, supporting a core collapse $\mathrm{SN}$ origin for RCW 86.

Acknowledgements. We thank the referee, K. Long, for useful comments on the manuscript, and J. Raymond, D. Cox and S. Reynolds for discussions This work was supported by the Swedish Research Council. PG acknowledges support from Chandra Grant GO0-1035X. $\mathrm{PL}$ is a Research Fellow at the Royal Swedish Academy supported by a grant from the Wallenberg Foundation.

\section{References}

Blair, W. P., Long, K. L., \& Vancura, O. 1991, ApJ, 366, 484 (BLV91) Blandford, R., \& Eichler, D. 1987, Phys. Rep., 154, 1

Boulares, A., \& Cox, D. P. 1988, ApJ, 333, 198

Brand, J., \& Blitz, L. 1993, A\&A, 275, 67

Chevalier, R. A., \& Raymond, J. C. 1978, ApJ, 225, L27

Chevalier, R. A., Kirshner, R. P., \& Raymond, J. C. 1980, ApJ, 235, 186

D’Odorico, S., Bandiera, R., Danziger, J., \& Focardi, P. 1986, AJ, 91, 1382

Ferriére, K. M. 2001, Rev. Mod. Phys., 73, 1031

Ghavamian, P. 1999, Ph.D. Thesis, Rice Univ.

Ghavamian, P., Raymond, J., Hartigan, P., \& Blair, W. P. 2000, ApJ, 535, 266 (GRHB00)

Ghavamian, P., Raymond, J., Smith, R. C., \& Hartigan, P. 2001, ApJ, 547, 995 (GRSH01)

Ghavamian, P., Winkler, P. F., Raymond, J., \& Long, K. S. 2002, ApJ, 572, 888 (GWRL02)

Ginzburg, V. L., \& Syrovatskii, S. I. 1964, The Origin of Cosmic Rays, trans. H. S. W. Massey, ed. D. ter Haar (New York: McMillan)

Hester, J. J., Raymond, J., \& Blair, W. P. 1994, ApJ, 420, 721 (HRB94)

Janev, R. K., Langer, W. D., Evans, K., Jr., \& Post, D. E., Jr. 1987, Elementary Processes in Hydrogen-Helium Plasmas (New York: Springer) 
Kirshner, R. P., Winkler, P. F., \& Chevalier, R. A. 1987, ApJ, 315, 589 Leibowitz, E. M., \& Danziger, I. J. 1983, MNRAS, 204, 273L Lim, A. J., \& Raga, A. C. 1996, MNRAS, 280, 103 Long, K. S, \& Blair, W. P. 1990, ApJ, 358, 13

Lyngå, G. 1964, Medd. Lund. Obs., Ser. II, 141, 1 Moffat, A. F. J., \& Reed, B. C. 1999, PASP, 111, 1149

Peimbert, M., \& van den Bergh, S. 1971, ApJ, 167, 223

Raymond, J. C., Blair, W. P., Fesen, R. A., \& Gull, T. R. 1983, 275, 636

Raymond, J. C. 1991, PASP, 103, 781

Raymond, J. C. 2001, Space Sci. Rev., 99, 209 (R01)
Rosado, M., Ambrocio-Cruz, P., Le Coarer, E., \& Marcelin, M. 1996, A\&A, 315, 243

Smith, R. C., Kirshner, R. P., Blair, W. P., \& Winkler, P. F. 1991, ApJ, 375, 652 (SKBW91)

Smith, R. C., Raymond, J. C., \& Laming, J. M. 1994, ApJ, 420, 286 (SRL94)

Smith, R. C. 1997, AJ, 114, 2664

Tuohy, I. R., Dopita, M. A., Mathewson, D. S., Long, K. S., \& Helfand, D. J. 1982, ApJ, 260, 65

Westerlund, B. E. 1969, ApJ, 74, 879

Winkler, P. F., \& Long, K. S. 1997, ApJ, 491, 829 